\title{
DIMENSIONAL ULTRASONOGRAPHIC RELATIONSHIP OF THE THYROID GLAND WITH ASSOCIATED ANATOMIC LANDMARKS IN CLINICALLY NORMAL DOGS
}

\author{
W.R. Bandula Kumara, N.O.D.M. Karunarathna and D.D.N. de Silva \\ Department of Veterinary Clinical Sciences, Faculty of Veterinary Medicine and Animal Science, University of \\ Peradeniya, Peradeniya, 20400, Sri Lanka.
}

\begin{abstract}
SUMMARY: Estimation of the size of the thyroid gland is considered to be important for diagnosis of several pathologic conditions in animals including dogs. Two-dimensional ultrasonography is used as a noninvasive simple method for determination of the size of thyroid gland. The purpose of the present study was to investigate the relationship among the dimensions of the thyroid gland with that of the associated anatomic landmarks of clinically normal dogs using ultrasonography. Ultrasonographic examinations were performed on 15 mixed breed dogs using an ultrasound scanner (MyLab30vet, Esaote, Genoa, Italy) with a linear-array transducer (Esaote LA 522, Esaote, Genoa, Italy). The maximal diameter and the length of the thyroid lobe were compared with that of the diameter of the common carotid artery and the trachea. The correlation between each thyroid parameter and the dimensions of the anatomic landmarks were assessed using linear regression analysis and Pearson's correlation coefficient test. According to the results, a significant linear correlation was observed between the maximum diameter of the thyroid lobe and the diameter of common carotid artery $(P=0.02$, Pearson's correlation coefficient $=0.188)$. The coefficient of determinations $\left(\mathbf{R}^{2}\right)$ for the maximum diameter of the thyroid lobe with that of common carotid artery diameter was 0.367 , indicating that the strengths of the association were weak. The mean ratio between the maximum thyroid lobe diameter and the common carotid artery diameter was $1.74 \pm 0.40$ (range $1.11-2.43$ ). However, there was no significant dimensional relationship with other selected anatomic landmarks. On the basis of the data obtained in this study, calculating the ratio between the maximum thyroid lobe diameter and the diameter of common carotid artery by using two-dimensional ultrasonography will be a useful and simple method for evaluating the size of the thyroid gland in dogs in clinical practice.
\end{abstract}

\section{INTRODUCTION}

The diagnosis of canine thyroid diseases is a challenging task to small animal clinicians. Clinical presentation is often nonspecific in canine thyroid diseases (Taeymans, 2009). The general diagnostic procedure of thyroid diseases in dogs consists of integrating patient history, physical examination results and thyroid profile testing (Taeymans, 2009). Estimation of the thyroid size is considered to be important in several pathologic conditions such as hyperthyroidism, hypothyroidism, neoplasia, and thyroiditis (Bromel et al., 2005; Reese et al., 2005; Taeymans et al., 2007; Vajhi et al., 2010; Ying et al., 2005). The size of the thyroid can be assessed by palpation, ultrasonography, scintigraphy, CT, and MRI examinations (Taeymans et al., 2008; Taeymans, 2009). However, Vajhi (2010) revealed that the evaluation of the size of the thyroid gland with palpation and scintigraphy was less accurate with errors between $82 \%$ and $90 \%$. Moreover, the techniques such as scintigraphy and $\mathrm{CT}$ require radiation, and thus are not suitable as routine examination modalities (Vajhi et al., 2010). On the other hand, use of biochemical methods, such as measuring resting serum tetraiodothyronine (T4) concentration to make the diagnosis of hypothyroidism makes it difficult to rule out euthyroid sick syndrome (Weber et al., 2000; Vitale et al., 2007). Furthermore, the biochemical methods which are specific to assess the thyroid function of dogs are not available in many countries.

The two-dimensional ultrasonography is a userfriendly, commonly available, noninvasive method for evaluation of the thyroid gland (Barberet et al., 2010; Bromel et al., 2005; Chaudhary et al., 2013; Taeymans et al., 2008; Wisner et al., 1998). Several anatomic landmarks, such as the medially located trachea, the laterally located common carotid artery, the ventrally located sternothyroid muscle, and the dorsally located esophagus in relation to the left lobe are often used for the identification of thyroid lobes during ultrasonographic evaluation (Taeymans, 2009). The left and right thyroid lobes are situated immediately caudal to the cricoid cartilage of the larynx, which serves as an external landmark for initial transducer placement (Taeymans, 2009).

In small animal clinical practice, several dimensional ultrasonographic relationships have been documented among various anatomic structures of the body (Mareschal et al., 2007; Rajapakshage et al., 2016), which can be used as rapid and simple diagnostic techniques. For example, the dimensional relationship between pancreas and the descending duodenum in clinically normal dogs has been reported (Rajapakshage et al., 2016). Likewise, Mareschal et al. (2007) has described the ultrasonographic measurement of kidney to abdominal aorta ratio as a method of estimating renal size in dogs. In a MRI study, 
Taeymans (2008) has revealed that the mean maximum thyroid lobe diameter on transverse images was almost twice the mean diameter of the common carotid artery. It has also been reported that the size of the thyroid gland is strongly related to the body weight of the dog (Bromel et al., 2006). However, to our knowledge, no ultrasonographic studies have been reported focused on investigation in to the dimensional correlation among the thyroid gland and associated anatomic landmarks of clinically normal dogs. The purpose of the present study was to examine the relationship among the ultrasonographic dimensions of the thyroid gland of dogs with those of the associated anatomic landmarks.

\section{MATERIALS AND METHODS Animals}

Ultrasonographic examinations were performed on 15 clinically normal mixed breed dogs ( 9 females and 6 males) between 1.2 years and 4.9 years of age (mean $2.8 \pm 0.3$ years) with body weights ranging from 9 to $18 \mathrm{~kg}$ (mean $12 \pm 0.6 \mathrm{~kg}$ ). Average body condition score was 2.5- 3.5 (on 1 - 5 scale). These dogs were presented to the Veterinary Teaching Hospital, University of Peradeniya for general checkups or elective surgical procedures, such as castration and ovariohysterectomy. A written consent from the owner was obtained before each ultrasonographic examination. General clinical examination was done on all animals. The concentration of T4 was measured in 6 dogs used in the study.

\section{Ultrasonography procedure}

Ultrasonographic examinations were performed using an ultrasound scanner (MyLab30vet, Esaote, Genoa, Italy) with a linear-array transducer (Esaote LA 522 , Esaote, Genoa, Italy) with frequency of $10 \mathrm{MHz}$. Ultrasonographic examinations were done over the ventral neck area with the dogs in dorsal recumbency. All the necessary dimensions were obtained from the sonographic images using electronic calipers. For each required parameter, at least three measurements were taken in millimeters, and the means of all measurements were calculated as the final value. Realtime images were recorded on videotape, and static images were sent to a computer equipped with specialized software for storage and off-line evaluation.

\section{Measuring the dimensions of the thyroid gland, the common carotid artery, and the trachea}

As described by Taeymans (2009), ultrasonographic examination was started in the mid ventral area of the neck caudal to the larynx. Firstly, the linear array transducer was placed transversely to obtain transverse plane images. The right and left thyroid lobes were confirmed between the common carotid arteries and the trachea immediately caudal to the cricoid cartilage on either side of the cervical region. The transverse view of thyroid lobe is more or less triangular in shape. The maximum thyroid lobe diameter was measured for each lobe, and then the average was calculated. Next, the transducer was rotated clockwise in 90 degrees to obtain the sagittal plane images of the thyroid lobe which is fusiform in shape. On the sagittal image, the length of the left and right thyroid lobes was measured and the average length was calculated.

The common carotid arteries were located on transverse sonograms lateral to the thyroid lobe on either side of the cervical region as anechoic circular structures with thick hyperechoic walls. Colour Doppler ultrasound was used to confirm the pulsating common carotid arteries. The diameters of both the left and right common carotid arteries were measured and the average was calculated. A ratio between the thyroid lobe diameter and the common carotid artery diameter was then obtained for each dog and the mean value was calculated.

The trachea was located in the mid cervical area. It was identified by its prominent tracheal rings which were cylindrical in shape with underling acoustic shadow and reverberation artefacts. The static image was obtained when trachea appears in horseshoe appearance. The diameter of the trachea was measured using the electronic caliper placed between the widest points of the trachea.

\section{Statistical analysis}

All measurements were recorded on a Microsoft Excel spreadsheet. Statistical analysis was done using the software Minitab. Descriptive statistics, such as mean, range, and standard deviations of the mean were calculated for all the measurements obtained. The average values were calculated using measurements obtained for both the left and right thyroid lobes and the common carotid artery. A ratio between the maximum thyroid lobe diameter and the common carotid artery diameter was then obtained. The normal distribution of the ratio was tested using Shapiro-Wilks test of normality. The correlation between thyroid measurements (maximum lobe diameter and length) and each parameter of the anatomical land marks (diameter of the common carotid artery and the trachea) were assessed using linear regression analysis and scatter plot. Similarly, the thyroid gland measurements were compared with other selected anatomic landmarks. The $p$ value $<0.05$ was considered as statistically significant.

\section{RESULTS}

A total of 15 mixed breed dogs were subjected to examination and the required ultrasonographic images were obtained. Mean heart rate of dogs used in the present study was $107.4 \pm 5.4 \mathrm{bpm}$ (range $90-120$ $\mathrm{bpm}$ ). The mean T4 level of 6 dogs used in the study was $21.8 \pm 7.4 \mathrm{nmol} / \mathrm{L}$ (reference range $11-60 \mathrm{nmol} / \mathrm{L}$ ).

The thyroid glands of all dogs appeared with homogeneous and hyperechoic parenchyma (Figures 1 and 2). Figure 1 represents the transverse cervical 
sonogram of a 3- year old mixed breed dog, showing the trachea, the left and right thyroid lobes, and the common carotid arteries, while Figure 2 illustrates the sagittal view of the left thyroid lobe of the same dog with anatomic relation to the carotid artery, the jugular vein, the esophagus, and the sternothyroid muscle.

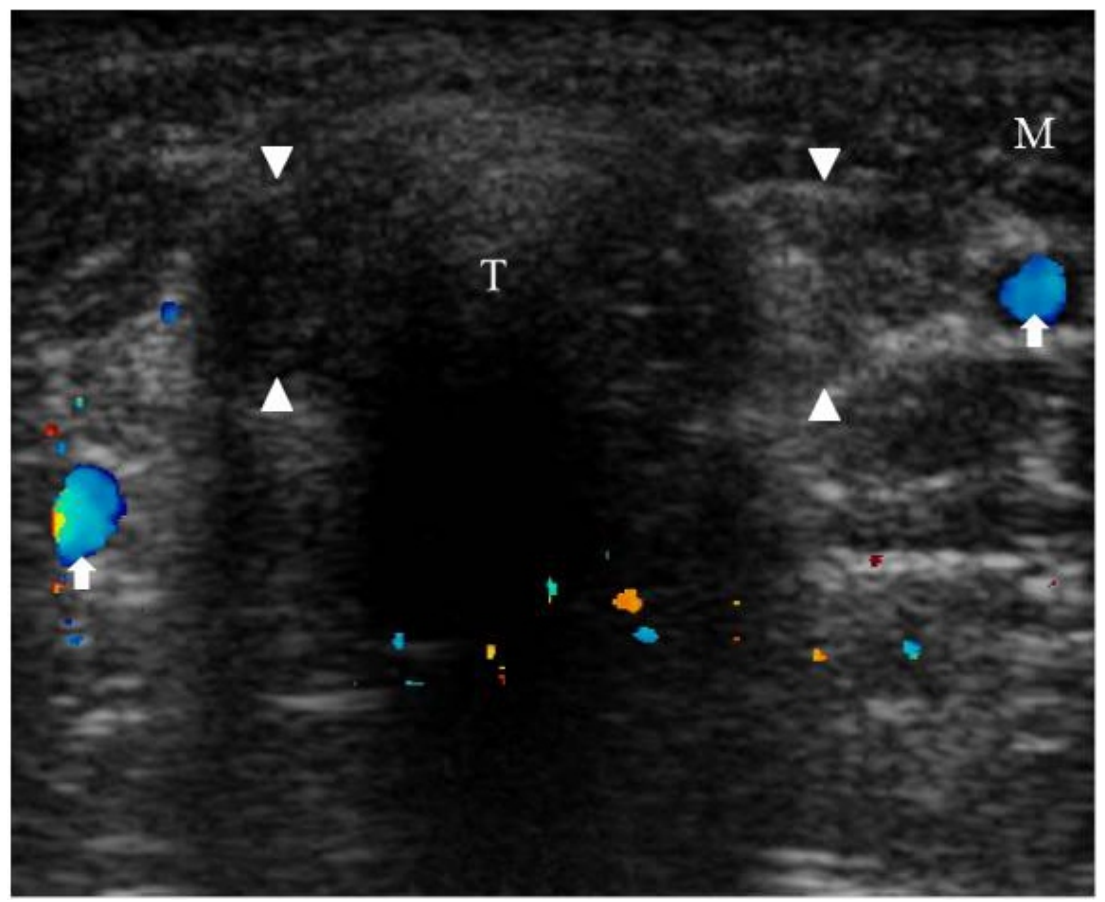

\section{Figure 1.}

Cervical sonogram of a 3 year old mixed breed dog showing transverse view of the left and right thyroid lobes (between white arrow heads) on either side of the trachea (T). The homogeneous thyroid lobe is triangular in shape an hyperechoic in comparison with the surrounding musculature (M). The left and right common carotid arteries can be seen (white arrows) lateral to each thyroid lobe. Ventral is to the top, dorsal is to the bottom, and right is to the left.

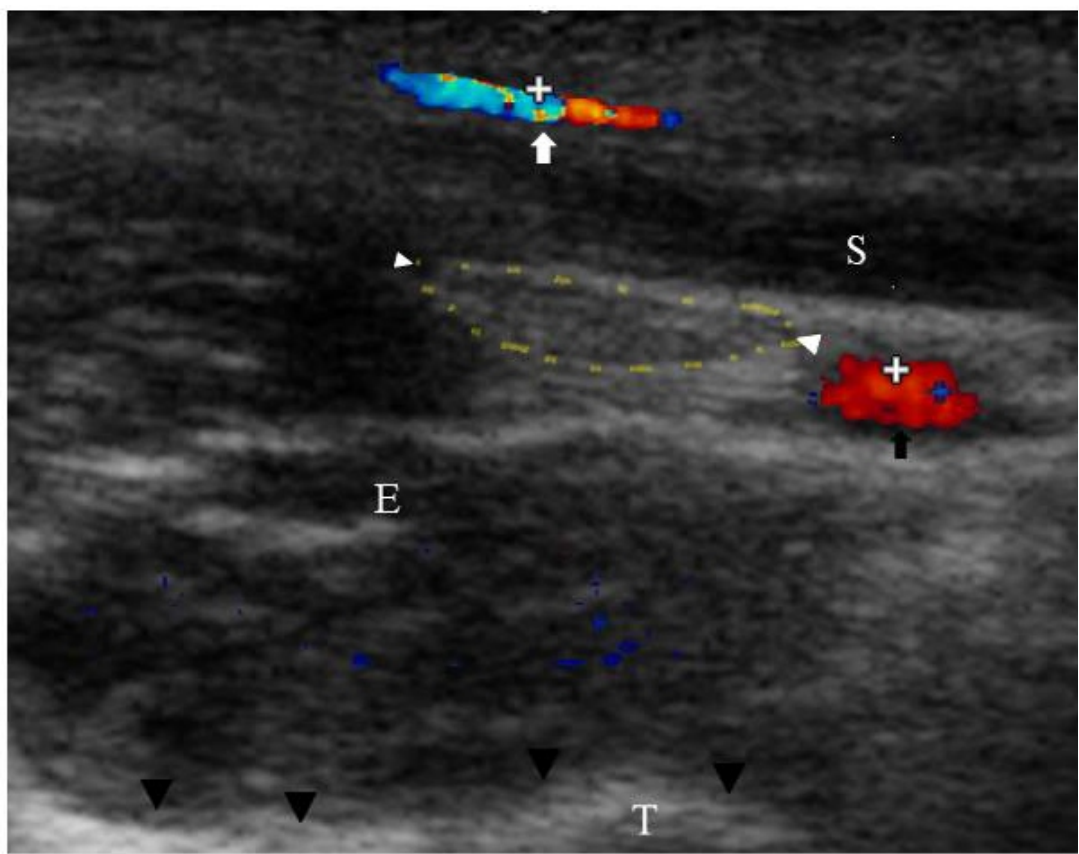

\section{Figure 2.}

Left lateral cervical sonogram of a 3 year old mixed breed dog showing the representative sagittal view of the left thyroid lobe (margined by dashed line) in fusiform shape. The length of the thyroid was measured between the two white arrow heads. The homogeneous thyroid lobe is hyperechoic in comparison with the ventrally located sternothyroid muscle (S) and the dorsally located esophagus (E). The common carotid artery (black arrow) and jugular vein (white arrow) can be seen. Black arrow heads direct at the left lateral margin of the trachea (T). Cranial is to the left, Caudal is to the right and Ventral is to the top. 
The mean maximum thyroid lobe diameter and length were $0.56 \pm 0.04 \mathrm{~cm}$ (range $0.29-1.00 \mathrm{~cm}$ ) and $2.16 \pm 0.10 \mathrm{~cm}$ (range $0.1 .48-2.78 \mathrm{~cm}$ ), respectively. The mean diameter of common carotid artery was 0.32 $\pm 0.01 \mathrm{~cm}$ (range $0.21-0.41 \mathrm{~cm}$ ). The mean tracheal diameter was $2.41 \pm 0.12 \mathrm{~cm}$ (range $1.55-3.06 \mathrm{~cm}$ ). As shown in Figure 3, the significant linear correlation $(P<0.05)$ was observed for the maximum thyroid lobe diameter with that of common carotid artery diameter $(P=0.02$, Pearson's correlation coefficient $=0.188)$. The coefficient of determinations $\left(\mathrm{R}^{2}\right.$ value) for the maximum thyroid lobe diameter with that of the common carotid artery diameter was 0.367 . The mean ratio between the maximum thyroid lobe diameter and the diameter of common carotid artery was $1.74 \pm 0.40$ (range $1.11-2.43$ ).

However, there was no dimensional relationship observed between the length of thyroid lobe and the diameter of common carotid artery $(P=0.3$, Pearson's correlation coefficient $=0.037$ ). In addition, there was no linear correlation between both the maximum thyroid lobe diameter with that of the diameter of trachea $(P=0.7$, Pearson's correlation coefficient $=$ -0.294) and length of thyroid lobe with that of the diameter of trachea $(P=0.5$, Pearson's correlation coefficient $=0.233$ ).

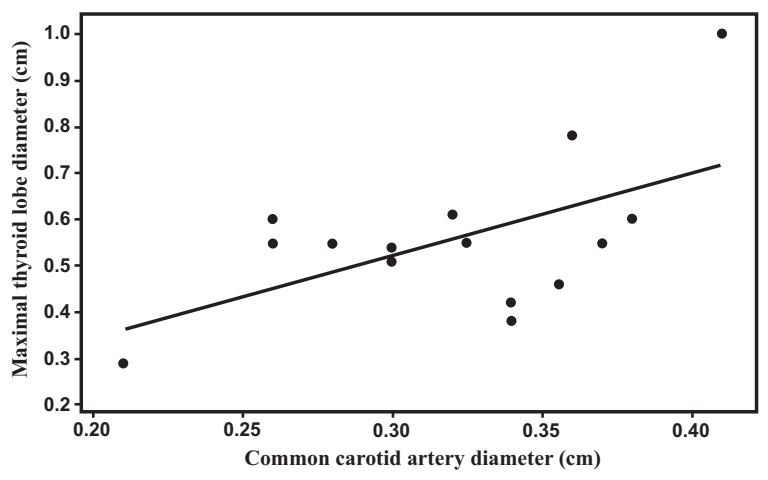

Figure 3.

The maximum thyroid lobe diameter as a function of diameter of common carotid artery, with the regression line. The regression equation is $\mathrm{y}=0.188 \mathrm{H}+0.217$, where $\mathrm{H}$ is the diameter of common carotid artery. The Pearson's correlation coefficient is 0.188 , with a $P$ value of 0.02 .

\section{DISCUSSION}

In this study, the dimensional ultrasonographic relationship of the thyroid gland with associated anatomic landmarks of clinically normal dogs was investigated. The thyroid lobe diameter showed a significant linear correlation with the diameter of the common carotid artery in clinically normal dogs. However, the coefficient of determinations of the association indicated that the strengths of the association were weak $\left(\mathrm{R}^{2}<0.5\right)$. The common carotid artery is a useful anatomic landmark which can be simultaneously imaged with the thyroid gland during ultrasonographic examinations in dogs (Barberet et al., 2010). Therefore, the ratio between the maximum thyroid lobe diameter and the diameter of common carotid artery $(1.74 \pm 0.40)$ can be used as a quick referral value in clinical practice. However, the ratio obtained in the present study was broader than the value reported in a previous MRI study (Taeymans et al., 2008). This is justifiable because the quality of ultrasonographic images is comparatively lower than the MRI images.

The canine thyroid gland is composed of two separate lobes. The right lobe is slightly more cranially positioned than the left one, and both lobes extend over a length from the cricoid cartilage to approximately the fifth to eighth tracheal ring (Taeymans et al., 2008). Although Figure 1 is used to illustrate both the left and right thyroid lobes in a single image, all the measurements were confirmed assessing both the left and right thyroid separately in each dog used in the present study.

There are few limitations that should be mentioned in the present study. In our study, all dogs were presumed to be free from thyroid diseases. The possibility of subclinical thyroid disorder, however, cannot be ruled out. The second drawback in our study was that serum biochemical analyses including canine specific thyroid function test could not be performed due to lack of test facilities in our clinical setting and due to their high cost. In addition, the range of body weights of 15 dogs was broad (range 9 to $18 \mathrm{~kg}$ ) in this study. Thus, it would have been better if the sample number could have been increased including various breeds of dogs.

In conclusion, there were weak but significant linear correlations between the maximum thyroid lobe diameter with the diameter of common carotid artery in clinically normal dogs. The measurements used to calculate the ratio were easy to obtain without complex reference tables. On the basis of the data obtained in this study, calculating the ratio between the maximum thyroid lobe diameter and the diameter of the common carotid artery with two-dimensional ultrasonography is a useful and simple method for evaluating the size of the thyroid gland of dogs in clinical practice.

\section{ACKNOWLEDGEMENTS}

The authors would like to thank the clinical staff at the Veterinary Teaching Hospital, Department of Veterinary Clinical Sciences, University of Peradeniya, Sri Lanka for their valuable assistance in carrying out this study. 


\section{REFERENCES}

Barberet, V. and Saunders, J.H.(2010). Ultrasonographic examination of selected small structures in dogs and cats : Thyroid glands, lymph nodes and adrenal glands. Vlaams Diergeneeskundig Tijdschrift, 79: $147-155$.

Bromel, C., Pollard, R.E., Kass, P.H., Samii, V.F., Davidson, A.P. and Nelson, R.W. (2006). Comparison of ultrasonographic characteristics of the thyroid gland in healthy small-, medium-, and large-breed dogs. American Journal of Veterinary Research, 67: 70-77. https://doi.org/10.2460/ajvr.67.1.70

Bromel, C, Pollard, R.E., Kass, P.H., Samii, V.F., Davidson, A.P. and Nelson, R.W. (2005). Ultrasonographic evaluation of the thyroid gland in healthy, hypothyroid, and euthyroid Golden Retrievers with nonthyroidal illness. Journal of Veterinary Internal Medicine, 19: 499-506.

htpps://doi.org/10.1111-j.1939-1676.2005.tb02718.x

Chaudhary, V. and Bano, S.(2013). Thyroid Ultrasound: Review article. Indian Journal of Endocrinology and Metabolism, 17: 219-227.

https://doi.org/10.4103/2230-8210.109667

Mareschal, A., Anjou, M.A., Moreau, M., Alexander, K. and Beauregard, G. (2007). Ultrasonographic measurement of kidney to abdominal aorta ratio as a method of estimating renal size in dogs. Veterinary Radiology Ultrasound, 48: 434-438.

https://doi.org/10.1111/j.1740-8261.2007.0027.x

Rajapakshage, B.K.W., Garuhamilage, J.P.K.E., de Silva, D.D.N. and Dangolla, A. (2016). Dimensional ultrasonographic relationship of the right lobe of pancreas with associated anatomic landmarks in clinically normal dogs. Journal of Veterinary Medical Science, 78:1-5.

https://doi.org/10.1292/jvms.15-0209

Reese, S., Breyer, U., Deeg, C., Kraft, W. and Kaspers, B. (2005). Thyroid sonography as an effective tool to discriminate between euthyroid sick and hypothyroid dogs. Journal of Veterinary Internal Medicine, 19: 491-498.

https://doi.org/10.1111/j.1939-1676.2005.tb027.x

Taeymans, O. (2009). Thyroid ultrasound in dogs : A review. Ultrasound, 17: 137-143.

https://doi.org/10.1179/174313409X448534

Taeymans, O., Dennis, R. and Saunders, J.H. (2008). Magnetic resonance imaging of the normal canine thyroid gland. Veterinary Radiology and Ultrasound, 49: 238-242. https://doi.org/10.1111/j.1740-8261.2008.00357.x

Taeymans, O., Daminet, S., Duchateau, L. and Saunders, J. H. (2007). Pre- and post-treatment ultrasonography in hypothyroid dogs. Veterinary Radiology and Ultrasound, 48: 262-269.

https://doi.org/10.1111/j.1740-8261.2007.00240.x

Vajhi, A. Rajabioun, M., Molazem1, M., Sharifi, H. and Vajhi, A. (2010). Ultrasonographic volumetry of the canine thyroid gland : A comparison of 2D, 3D ultrasonography and real gland volume. Iranian Journal of Veterinary Surgery, 5: 45-50.

Vitale, C.L. and Olby, N.J.(2007). Neurologic dysfunction in hypothyroid, hyperlipidemic Labrador Retrievers. Journal of Veterinary Internal Medicine, 21: $1316-1322$

https://doi.org/10.1111/j.1939-1676.2007.tb01954.x

Weber, A.L, Randolph, G. and Aksoy, F.G. (2000). The thyroid and parathyroid glands. CT and MR imaging and correlation with pathology and clinical findings. Radiologic Clinics of North America, 38: 1105-1129. https://doi.org/10.1016/S0033-8389(05)70224-4

Wisner, E.R. and Nyland, T.G. (1998). Ultrasonography of the thyroid and parathyroid glands. Veterinary Clinics of North America: Small Animal Practice, 28: 973-991.

https://doi.org/10.1016/S0195-5616(98)50085-X

Ying, M., Sin, M. and Pang, S. (2005). Sonographic measurement of thyroid gland volume: A comparison of 2D and 3D ultrasound. Radiography, 11: 242-248 https://doi.org/10.1016/j.radi.2005.03.010 